\title{
Hábitat de anidación de Amazona oratrix (Psittaciformes: Psittacidae) en el Pacífico Central, México
}

\author{
Tiberio C. Monterrubio-Rico ${ }^{1}$, Margarito Álvarez-Jara ${ }^{1}$, Lorena Téllez-García ${ }^{1}$ \& \\ Carlos Tena-Morelos ${ }^{2}$
}

1. Laboratorio de Ecología de Vertebrados Terrestres Prioritarios, Facultad de Biología, Universidad Michoacana de San Nicolás de Hidalgo. Avenida Francisco J. Mújica S/N, Ciudad Universitaria, Colonia Felícitas del Río, Morelia, Michoacán, 58030, México; tmonter2002@yahoo.com.mx, alvarez_jara_m@hotmail.com, lorpa19@yahoo.com

2. Laboratorio de Agroecología, Facultad de Biología, Universidad Michoacana de San Nicolás de Hidalgo. Avenida Francisco J. Mújica S/N, Ciudad Universitaria, Colonia Felícitas del Río, Morelia, Michoacán, 58030, México; carlostena56@hotmail.com

\author{
Recibido 04-XI-2013. Corregido 18-II-2014. Aceptado 13-III-2014.
}

\begin{abstract}
Nesting habitat characterization for Amazona oratrix (Psittaciformes: Psittacidae) in the Central Pacific, Mexico. The nesting requirements of the Yellow-headed Parrot (Amazona oratrix) are poorly understood, despite their broad historical distribution, high demand for pet trade and current endangered status. Information concerning their nesting requirements is required in order to design specific restoration and conservation actions. To assess this, we studied their nesting ecology in the Central Pacific, Michoacan, Mexico during a ten year period. The analyzed variables ranged from local scale nest site characteristics such as nesting tree species, dimensions, geographic positions, diet and nesting forest patches structure, to large scale features such as vegetation use and climatic variables associated to the nesting tree distributions by an ecological niche model using Maxent. We also evaluated the parrot tolerance to land management regimes, and compared the Pacific nest trees with 18 nest trees recorded in an intensively managed private ranch in Tamaulipas, Gulf of Mexico. Parrots nested in tall trees with canopy level cavities in 92 nest-trees recorded from 11 tree species. The $72.8 \%$ of nesting occurred in trees of Astronium graveolens, and Enterolobium cyclocarpum which qualified as keystone trees. The forests where the parrots nested, presented a maximum of 54 tree species, $50 \%$ of which were identified as food source; besides, these areas also had a high abundance of trees used as food supply. The lowest number of tree species and trees to forage occurred in an active cattle ranch, whereas the highest species richness was observed in areas with natural recovery. The nesting cavity entrance height from above ground of the Pacific nesting trees resulted higher than those found in the Gulf of Mexico. We hypothesize that the differences may be attributed to Parrot behavioral differences adapting to differential poaching pressure and cavity availability. Nesting trees were found in six vegetation types; however the parrots preferred conserved and riparian semi-deciduous forest for nesting, with fewer nests in deciduous forest, while nesting in transformed agricultural fields was avoided. The main climatic variables associated with the potential distribution of nests were: mean temperature of wettest quarter, mean diurnal temperature range, and precipitation of wettest month. Suitable climatic conditions for the potential presence of nesting trees were present in $61 \%$ of the region; however, most of the area consisted of tropical deciduous forests (55.8\%), while semi-deciduous tropical forests covered only $17 \%$ of the region. These results indicated the importance to conserve semi-deciduous forests as breeding habitats for the Yellow-headed Parrot, and revealed the urgent need to implement conservation and restoration actions. These should include a total ban of land use change in tropical semi-deciduous forest areas, and for selective logging of all keystone tree species; besides, we recommend the establishment of wildlife sanctuaries in important nesting areas, and a series of tropical forest restoration programs in the Central Pacific coast. Rev. Biol. Trop. 62 (3): 1053-1072. Epub 2014 September 01.
\end{abstract}

Key words: nesting habitat, diet composition, climatic conditions. 
El loro cabeza-amarilla (Amazona oratrix Ridgway 1887) es una de las especies de mayor tamaño (35.5-38cm) del género Amazona. Su mayor área de distribución se encuentra en México, y parte de Belice, con algunos registros para Guatemala y Honduras; así, tres de las cuatro subespecies reconocidas son prácticamente endémicas de México: $A$. oratrix magna en el Golfo de México, A. oratrix oratrix en la costa del Pacífico, y A. oratrix tresmariae limitada al archipiélago de las Islas Marías (Howell \& Webb, 1995; Collar et al., 2000). Esta especie, anida obligadamente en cavidades secundarias (Monterrubio-Rico \& Escalante-Pliego, 2006), y una cavidad que es utilizada se reconoce por la frecuente actividad de loros cerca de ella (Enkerlin-Hoeflich, 1995; Téllez-García, 2008). Se ha documentado que en el Pacífico las oquedades usadas como nidos tienen de 15 a $28 \mathrm{~cm}$ de diámetro interno y una profundidad de 41 a $51 \mathrm{~cm}$; en tanto que el Golfo de México se ha observado un diámetro interno de 14 a $52 \mathrm{~cm}$, y una profundidad de 51 a 260 cm (Enkerlin-Hoeflich, 1995; Téllez-García, 2008). En términos generales, el hábitat del loro cabeza-amarilla ha incluido bosques tropicales subcaducifolios húmedos $\mathrm{y}$ subhúmedos, caducifolios secos, de ribera, y arboledas en áreas ganaderas (Ridgely, 1981; Enkerlin-Hoeflich, 1995; Howell \& Webb, 1995; Stotz, Fitzpatrick, Parker III \& Moskovits, 1996; Collar et al., 2000). En México, el loro cabeza-amarilla presentaba una distribución histórica relativamente extensa, desde tierras bajas tropicales de ambas vertientes (Golfo de México y Pacífico) hasta los 900msnm (Forshaw, 1989; Howell \& Webb, 1995), estimada en 150 830km² (Ríos-Muñoz \& NavarroSigüenza, 2009). En el Pacífico de México, habita en paisajes donde predomina el bosque tropical caducifolio, seguido en menor cobertura por el bosque tropical subcaducifolio, y en creciente porcentaje las áreas agropecuarias y de desarrollos turísticos (Monterrubio-Rico, Renton, Ortega-Rodríguez, Pérez-Arteaga \& Cancino-Murillo, 2010).

Sin embargo, la expansión de la ganadería, la tala selectiva, y los incendios, han reducido la extensión y han degradado los bosques tropicales existentes en ambas vertientes. En la costa central del Pacífico de México, las áreas donde existían bosques tropicales subcaducifolios han sido degradadas a pastizales para ganadería, agricultura y plantaciones de frutales, a una tasa anual estimada de $0.65 \%$ (Mas-Caussel, Velásquez-Montes \& Fernández-Vargas, 2005; Monterrubio-Rico et al., 2010). La pérdida y degradación de bosques tropicales en las tierras bajas tuvo consecuencias drásticas para el loro cabeza-amarilla, estimándose una pérdida hipotética de $58.9 \%$ del hábitat potencial para la especie (Ríos-Muñoz \& Navarro-Sigüenza, 2009). En el Pacífico, se estima que ya sólo se distribuye en el $21 \%$ de su distribución histórica (Monterrubio-Rico et al., 2010). Incluso en sitios como la costa de Michoacán, el loro cabeza-amarilla desapareció de áreas con vegetación conservada (probablemente debido al saqueo de nidos) perdiéndose así el 54\% de su distribución (Monterrubio-Rico et al., 2007).

El declive en el tamaño de sus poblaciones y la drástica reducción de su distribución fueron los principales argumentos para sustentar que la especie fuera considerada amenazada a nivel global (Collar et al., 2000; Snyder, McGowan, Gilardi, \& Grajal, 2000; CantúGuzmán, Sánchez-Saldaña, Grosselet, \& SilvaGámez, 2007; Monterrubio-Rico et al., 2010; IUCN, 2013), y en peligro de extinción en México (SEMARNAT, 2010). A pesar de su condición de amenaza, existe poca información científica publicada y disponible sobre sus requerimientos de hábitat, sitios de anidación y dieta. La información acerca de los requerimientos para su reproducción se limita a un par de trabajos en áreas bajo condiciones muy particulares. En el Golfo de México, el único estudio publicado reportó a la especie anidando de enero a junio en cavidades de Bumelia laetevirens, Pithecellobium ebano y Ficus cotinifolia en un rancho ganadero (Enkerlin-Hoeflich, 1995). En la vertiente del Pacífico, la población remanente más norteña de loro cabezaamarilla (Monterrubio-Rico et al., 2010), anida en la Reserva de la Biosfera de ChamelaCuixmala desde febrero a junio en cavidades 
de Astronium graveolens, Bursera arborea, y Sideroxylon palmeri (Renton, 2002).

Ante la pérdida de distribución que ha sufrido la especie (Monterrubio-Rico et al., 2007), y ante la falta de estimaciones poblacionales actuales, es necesario conocer más detalladamente sus requerimientos de anidación. Adicionalmente, se debe generar información con respecto a las características a nivel local como las de sus árboles-nido, hasta las condiciones climáticas de aptitud a nivel regional de las áreas donde anida, incluyendo los tipos de vegetación, y las condiciones de manejo que toleran; esta es información esencial para diseñar acciones concretas de conservación y manejo, así como para el establecimiento de vedas, selección de áreas de conservación, o sitios prioritarios para restaurar.

Por lo anterior, en este estudio presentamos información actual sobre la ecología de anidación de la especie, desde las características a escala local como los árboles-nido, su ubicación, la estructura de las áreas de bosques donde anida, y se alimenta, así como las condiciones de manejo que tolera. A escala de paisaje, generamos un modelo de distribución potencial para delimitar las áreas con condiciones climáticas adecuadas para la anidación e identificamos las principales variables climáticas asociadas. Esta información es indispensable para diseñar las acciones específicas de protección y restauración del hábitat, necesarias para conservar una población reproductora de la especie en el Pacífico central de México.

\section{MATERIALES Y MÉTODOS}

Región de estudio: Incluyó los municipios de Aquila, Lázaro Cárdenas y Arteaga en la región sierra-costa central del Pacífico en Michoacán, con una extensión de $6925 \mathrm{~km}^{2}$ (Fig. 1). La región presenta una estrecha planicie costera $(3 \mathrm{~km})$, con topografía que incluye colinas bajas $(230 \mathrm{msnm})$ hasta cordilleras con
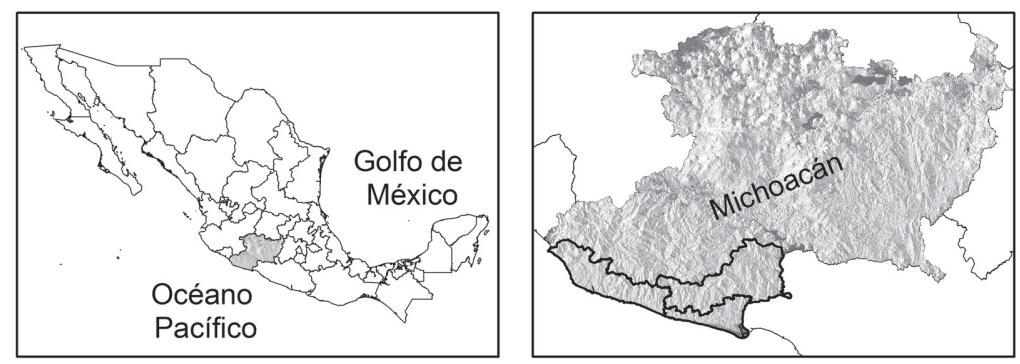

Simbología
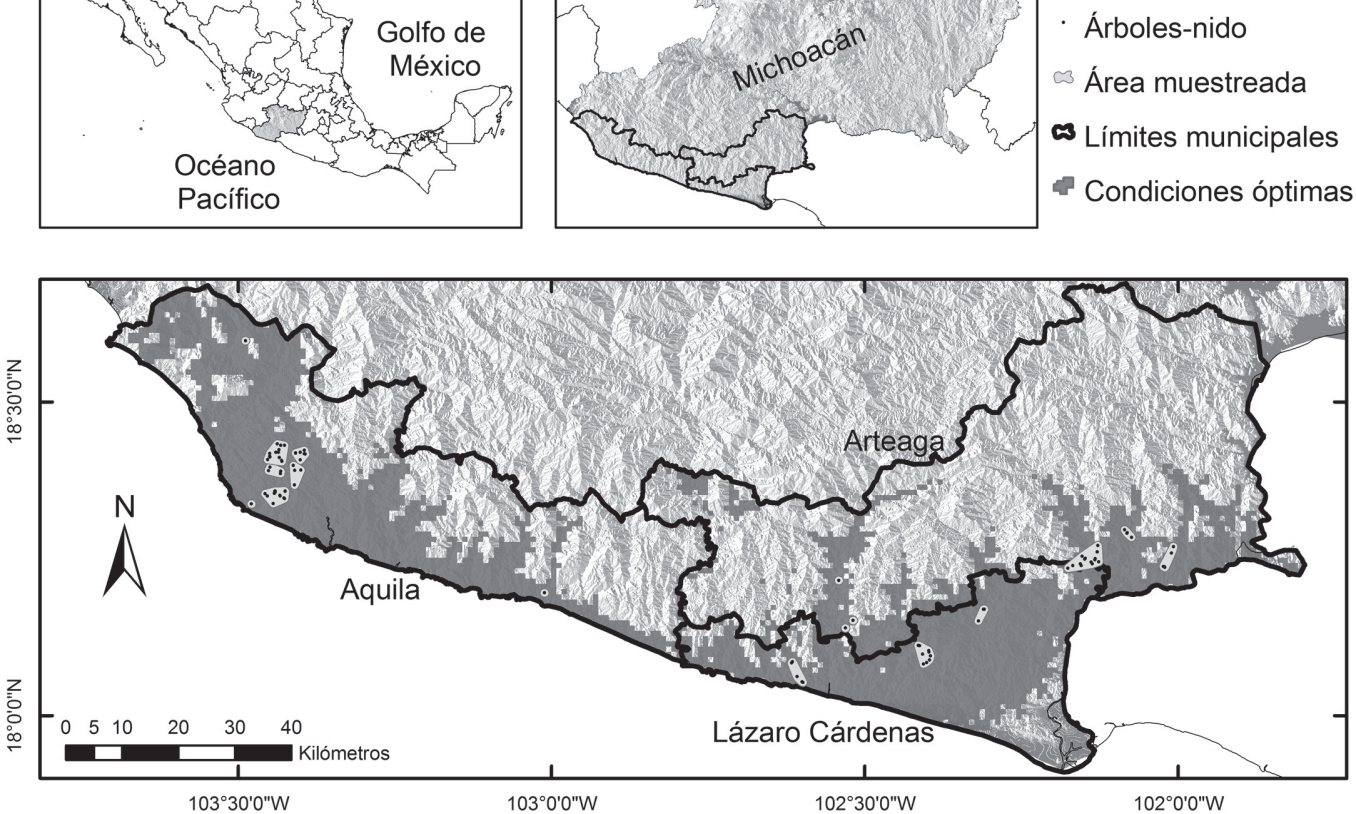

Fig. 1. Áreas con condiciones climáticas adecuadas para la distribución potencial de nidos de loro cabeza-amarilla.

Fig. 1. Suitable climatic area for the potential distribution of Yellow-headed Parrot nest. 
elevaciones superiores a $1000 \mathrm{msnm}$, donde a través de cañadas y barrancas fluyen hacia el Océano Pacífico 52 ríos, creando bosques en condición de ribera y microambientes más húmedos.

La vegetación predominante de la región es el bosque tropical caducifolio, que forma comunidades densas con una altura promedio de entre 8 y $12 \mathrm{~m}$. Sus especies dominantes incluyen Amphypteringium adstringens, Cyrtocarpa procera, Tabebuia palmeri, Lysiloma acapulcensis, Pseudobombax ellipticum, así como cactáceas columnares Stenocereus sp. y especies del género Bursera (CarranzaGonzález, 2005). Este tipo de bosque se desarrolla en suelos secos y rocosos, en colinas y laderas escarpadas, y cubre el 23\% (1 560 $\left.\mathrm{km}^{2}\right)$ del territorio, y donde el $25 \%$ de esta extensión se encuentra en condiciones perturbadas (SEMARNAP-UNAM, 2000; INEGI, 2009).

El segundo tipo de vegetación de la región es el bosque tropical subcaducifolio, caracterizado por presentar un estrato arbóreo alto (15 a $30 \mathrm{~m}$ ). Este tipo de bosque ocupa las áreas más húmedas desde el nivel del mar hasta los 1000msnm (Carranza-González, 2005). Las especies dominantes incluyen: Brosimum alicastrum, Bursera simaruba, Astronium graveolens, Bumelia persimilis, Cedrela odorata, Cordia elaeagnoides, Dalbergia granadillo, Hura poliandra y varias especies de Ficus. Este tipo de bosque no presenta grandes extensiones continuas, se presenta a manera de secciones de tamaño irregular a lo largo de barrancas, márgenes de ríos, y laderas sombreadas. El bosque tropical subcaducifolio conservado mantiene sólo el $7 \%\left(454 \mathrm{~km}^{2}\right)$ de la región, en condición perturbada un $6 \%\left(413 \mathrm{~km}^{2}\right)$, y en condición de ribera el $2 \%\left(107.7 \mathrm{~km}^{2}\right)$. Las áreas con agricultura y ganadería cubren el $38 \%$ de la superficie de la región (SEMARNAP-UNAM, 2000; INEGI, 2009).

Trabajo de campo y caracterización de los árboles-nido: Se estudió la actividad de anidación del loro cabeza-amarilla desde finales de enero hasta principios de mayo desde el 2002 al 2013. Debido a limitaciones logísticas y de inseguridad, sólo se estudió detalladamente la composición del estrato arbóreo alrededor de los nidos presentes en tres localidades ( $\mathrm{La}$ Bonetera, El Habillo, y Motín del Oro; 20062007). Para comparar las proporciones de uso y disponibilidad de cada tipo de vegetación, sólo se analizaron cinco localidades en las que se verificó en campo la extensión de cada tipo de vegetación (La Bonetera, El Habillo, Motín del Oro, Chuquiapan, y Arteaga; 2003-2009). Aunque se dispone de cartografía digital de vegetación basada en imágenes de satélite, existen inconsistencias entre la vegetación que localmente se observa, y la de la cartografía. La compleja topografía crea un mosaico de vegetación con ecotonos donde pequeños fragmentos de vegetación, especialmente de bosque tropical subcaducifolio y los parches en condición de ribera ( $\leq 5 \mathrm{~h} a \mathrm{~s})$, no aparecen en los mapas digitales ya que no se pueden cartografiar, por lo tanto, no incluimos en estos análisis aquellas áreas de anidación donde no se pudo verificar en campo la extensión de cada tipo de vegetación.

En la búsqueda y ubicación de nidos de loro cabeza-amarilla se contó con la ayuda de habitantes locales de 20 a 50 años como guías y apoyo logístico, quienes son campesinos, y/o cazadores, y además fueron comisionados por las autoridades locales para apoyar en la investigación.

La búsqueda de nidos se efectuó durante enero, previo a la puesta de huevos, aunque algunos nidos se encontraron entre marzo y abril. Los árboles-nido se ubicaron observando el comportamiento de parejas de loros desde sitios elevados, y revisando árboles en los que los habitantes locales reportaban haber visto a los loros. Una cavidad fue considerada como nido potencial si algún miembro de la pareja de loros ingresaba y duraba más de $30 \mathrm{~min}$ en ella, o si se observó entrar más de una vez en un día durante el período previo a la puesta. Durante la etapa de cría (última semana de febrero y principios de marzo), las cavidades fueron confirmadas como nidos cuando los loros adultos entraron varias veces al día, y exhibían conducta de alimentación (Enkerlin-Hoeflich, 
1995; Téllez-García, 2008). Adicionalmente, los árboles accesibles fueron revisados por guías locales autorizados por la comunidad, para verificar el contenido de la cavidad sin dañarla, con base en usos y costumbres. Cada nido encontrado fue revisado en los años subsecuentes dependiendo de la capacidad logística, y cada árbol-nido fue caracterizado midiendo las siguientes variables: especie, condición (vivo o muerto), diámetro a la altura del pecho (DAP), altura de la cavidad desde el suelo, número de cavidades, altura del árbol, orientación de cavidad, y las coordenadas geográficas.

Se utilizó el índice de amplitud del nicho de Levins estandarizado para evaluar que tan generalista o estricto es el loro en el uso de especies de árboles para anidar; para este índice, valores cercanos de 0 significaron especialización, y los cercanos a 1 indicaron mayor amplitud (Levins, 1968; Colwell \& Futuyma, 1971; Krebs, 1989).

Se usó el sistema de información geográfica Arc Map Version 9.3 y sus módulos y herramientas (Spatial Analyst, Hawth's Tools, Land Facet Corridor Designer; Beyer, 2004; ESRI, 2008; Jenness, Brost, \& Beier, 2012) para delimitar las zonas de anidación estudiadas, y estimar la extensión de cada tipo de vegetación en la región. También para definir características geográficas de los árboles-nido, como altitud, orientación de ladera, pendiente, y posición topográfica, así como para estimar su distancia a poblados, carreteras, arroyos y ríos.

Se compararon las características (altura y DAP) de árboles-nido de Michoacán, con las de 18 árboles-nido registrados en la planicie costera de Tamaulipas en el Golfo de México (Enkerlin-Hoeflich, 1995) empleándose la prueba de Mann-Whitney, con la finalidad de conocer la consistencia de la especie en la selección de características de los sitios para anidar.

\section{Estructura de la comunidad arbórea:} Durante el periodo 2006-2008, se identificó la composición arbórea alrededor de 16 árbolesnido localizados en tres áreas que difieren en manejo y régimen de propiedad. Las áreas fueron "El Habillo" (ganadería y pequeña propiedad privada; 400has), "La Bonetera" (regeneración natural y pequeña propiedad privada; 700has), y Motín del Oro (extracción selectiva con manejo comunal indígena nahua; 54448.6has). En estas áreas se identificaron y midieron todos los árboles con un $\mathrm{DAP} \geq 10 \mathrm{~cm}$ en parcelas circulares de $25 \mathrm{~m}$ de radio (área $=1963.6 \mathrm{~m}^{2}$ ) centradas en los árboles-nido (Marsden \& Jones, 1997; Marsden \& Pilgrim, 2003). Para identificar las especies de árboles se utilizaron las claves de Pennington y Sarukhán (2005). Adicionalmente, se generó una lista de las especies utilizadas como alimento, incluyendo observaciones en campo, entrevistas y revisión de literatura (EnkerlinHoeflich, 1995; Renton, 2002; Fabián-Turja, 2004; Morales-Pérez, 2005; Téllez-García, 2008; Delgado-Carrillo, 2012).

Se utilizaron pruebas no paramétricas (Kruskal-Wallis) para comparar las dimensiones de los árboles entre los distintos tipos de vegetación. La estadística descriptiva se presenta con medias, desviaciones estándar y/o rangos. Todas las pruebas y los estadísticos descriptivos se realizaron utilizando diferentes módulos de SAS (SAS Institute Inc., 2001). Se consideró el nivel de $\alpha$ de $\mathrm{p}<0.05$ (Zar, 1996).

Hábitats de anidación: Para conocer si existen preferencias o rechazo por algún tipo de vegetación en particular para anidar por parte del loro en la costa del Pacífico, se compararon las proporciones de nidos (43 nidos) encontrados en cinco localidades (El Habillo, La Bonetera, Motín del Oro, Chuquiapan y Arteaga) por varios años, por tipo de vegetación, con las proporciones de cada tipo de vegetación disponible en las áreas de estudio, empleando la prueba $\mathrm{G}$ de independencia, e intervalos de confianza de Bonferroni (Neu, Byers, \& Peek, 1974; Byers, Steinhorst, \& Krausman, 1984). El tipo de vegetación donde se localizó cada nido se definió considerando la vegetación predominante en un lote circular de $50 \mathrm{~m}$ de radio, ya que los nidos en ocasiones se localizaron en ecotonos. Los criterios para asignar la condición (conservado-perturbado) de la vegetación fueron los siguientes: 1) bosque conservado: 
si todo el lote contaba con vegetación sin evidencia de tala o claros en el bosque, 2) bosque perturbado: si la vegetación ocupaba $\leq 50 \%$ de la parcela que equivale $<1$ ha (Trejo \& Dirzo, 2000), y 3) tierras agrícolas transformadas: si el árbol-nido ocurrió en área agrícola o pastizal. El tipo de vegetación se asignó como bosque tropical caducifolio, subcaducifolio, o subcaducifolio ribereño si se presentaba en el cauce de arroyos o ríos, siguiendo la clasificación general de Rzedowski (1994).

La vegetación se comparó espacialmente con la reportada por el Inventario Forestal Nacional 2000, con mapas digitales actualizados con la serie IV (INEGI, 2009); para calcular las proporciones de los tipos de vegetación donde se localizaron los nidos, y su disponibilidad en las áreas. Todos los tipos de agricultura, horticultura y pastizales se reclasificaron y agruparon como superficie de uso agropecuario.

Características climáticas: Se estimó y delimitó la superficie de la región con aptitud para la presencia potencial de nidos, identificando también las variables climáticas asociadas a la distribución potencial usando Maxent. Este algoritmo proporciona curvas de respuesta de las distintas variables ambientales a los registros y estima la importancia de cada una en la distribución de la especie (Phillips, Dudík \& Schapire, 2004; Phillips, Anderson, \& Schapire, 2006). En el proceso de modelación se utilizaron 92 coordenadas de árboles-nido registrados y 19 variables climáticas (temperatura y precipitación) obtenidas de Worldclim (www.worldclim.org). Se programó el algoritmo para que internamente considerara el 100\% de los datos para generar el modelo. El modelo de salida se reclasificó utilizando como valor de corte el del umbral "Minimum training presence", debido a que este presenta los valores más bajos de omisión y la menor superficie de presencia, lo cual elimina los errores de omisión en el modelo, obteniéndose un mapa binario con áreas de presencia de condiciones de aptitud y áreas sin condiciones.

La evaluación de los modelos se hizo con el método de "ROC parcial", que ha demostrado ser efectivo al ponderar los errores de omisión, no considerar las ausencias, y evaluar sólo en el rango de valores de predicción (Peterson, Papes \& Soberón, 2008). Se usó el programa Tool for Partial-ROC versión 1.0 (Narayani, 2008), que hace un análisis de "Bootstrap" con los datos de presencia asociados a los valores de adecuación del modelo, obteniéndose el promedio del valor de radio de las iteraciones para después calcular la probabilidad de que el valor sea menor o igual a 1 , mediante una prueba de $z$. De acuerdo con Peterson et al. (2008), se consideran buenos modelos, aquellos cuyos valores promedio de radio fueran mayores a 1 y con probabilidad de 0 .

\section{RESULTADOS}

Especies de árboles-nido: Se localizaron 92 árboles-nido de loro cabeza-amarilla en 15 localidades o propiedades de tres municipios (Aquila $n=53$, Arteaga $n=27$, y Lázaro Cárdenas $n=12$ ). El área total muestreada fue de $119.7 \mathrm{~km}^{2}$ (Aquila $64.4 \mathrm{~km}^{2}$, Arteaga $38.7 \mathrm{~km}^{2}$, Lázaro Cárdenas $\left.16.6 \mathrm{~km}^{2}\right)$. Los nidos se localizaron en diferentes años: 9 entre 2002 y 2003, 27 entre 2005-2013, 24 entre 2007-2009, 3 entre 2007-2010, y 29 entre 2012-2013 (Fig. 1).

Los nidos se localizaron en 11 especies de árboles, con 52\% de los nidos ubicados en dos especies del bosque tropical subcaducifolio (Astronium graveolens y Brosimum alicastrum), aunque un porcentaje elevado (29.3\%; $\mathrm{n}=27)$ se encontró en Enterolobium cyclocarpum, árbol que forma parte tanto de bosque tropical subcaducifolio, como de ecotonos con bosque tropical caducifolio. Otras especies fueron: Tabebuia rosea $(\mathrm{n}=5)$, Cordia elaeagnoides $(\mathrm{n}=3)$, Licania arborea $(\mathrm{n}=3)$ y una especie $(\mathrm{n}=2)$ que probablemente es Aspidosperma megalocarpon. Un nido fue localizado en cada una de las siguientes especies: Piranhea mexicana, Dalbergia granadillo, Lonchocarpus sp. y Tabebuia sp. (Cuadro 1). La mayoría de los nidos se encontraron en árboles vivos $(\mathrm{n}=85$, 92.4\%). Con base en el índice de amplitud de nicho de Levins, el loro cabeza-amarilla 
CUADRO 1

Características principales de las especies de árboles-nido y su distribución por tipo de vegetación

TABLE 1

Species nest trees, their DHB and height mean, \pm standard deviation (range) and vegetation types where these occurred

\begin{tabular}{|c|c|c|c|c|c|c|c|c|c|}
\hline Especie & $n(\%)$ & $\mathrm{DAP}(\mathrm{cm})$ & $\begin{array}{c}\text { Altura de } \\
\text { cavidad (m) }\end{array}$ & BTC & ВТCP & BTS & BTSP & $\mathrm{BR}$ & $\begin{array}{l}\text { AB-BTS } \\
(\leq 0.5 \text { ha })\end{array}$ \\
\hline Astronium graveolens & $40(43.5)$ & $49.8 \pm 10.8(36-80)$ & $14.6 \pm 4.5(7-23)$ & 4 & 1 & 9 & 5 & 6 & 15 \\
\hline Enterolobium cyclocarpum & $27(29.3)$ & $115.8 \pm 45.3(40-195)$ & $14.6 \pm 3.4(7-20)$ & 1 & 1 & 6 & 1 & 15 & 3 \\
\hline Brosimum alicastrum & $8(8.7)$ & $78.4 \pm 18.3(41-99)$ & $16.1 \pm 5.3(8-23)$ & - & - & 6 & 2 & - & - \\
\hline Tabebuia rosea & $5(5.4)$ & $55.8 \pm 10.3(40-68)$ & $13.4 \pm 3.4(10-18)$ & - & 1 & - & - & 4 & - \\
\hline Cordia eleagnoides & $3(3.3)$ & $42.7 \pm 8.1(38-52)$ & $10.3 \pm 4.9(7-16)$ & 2 & - & - & - & - & 1 \\
\hline Licania arborea & $3(3.3)$ & $80.0 \pm 11.3(67-87)$ & $19.3 \pm 3.2(17-23)$ & - & - & 1 & - & 1 & 1 \\
\hline Aspidosperma megalocarpon & $2(2.2)$ & $40 \pm 0(40-40)$ & $11 \pm 1.4(10-12)$ & - & - & 1 & - & - & 1 \\
\hline Piranhea mexicana & $1(1.1)$ & 50 & 18 & - & - & - & 1 & - & - \\
\hline Dalbergia granadillo & $1(1.1)$ & 50 & 15 & - & 1 & - & - & - & - \\
\hline Lonchocarpus sp. & $1(1.1)$ & 38 & 20 & - & - & 1 & - & - & - \\
\hline Tabebuia sp. & $1(1)$ & 50 & 15 & - & - & - & - & 1 & - \\
\hline TOTALES & 92 & $72.4 \pm 39.5(36-195)$ & $14.7 \pm 4.2(7-23)$ & 7 & 4 & 24 & 9 & 27 & 21 \\
\hline
\end{tabular}

$\mathrm{BTC}=$ Bosque tropical caducifolio; $\mathrm{BTCP}=$ Bosque tropical caducifolio perturbado; $\mathrm{BTS}=$ Bosque tropical subcaducifolio; $\mathrm{BTSP}=\mathrm{Bosque}$ tropical subcaducifolio perturbado, $\mathrm{BR}=\mathrm{B}$ osque de ribera $\mathrm{AB}-\mathrm{BTS}=$ Arboledas de bosque tropical subcaducifolio.

presentó una estrecha amplitud de nicho para el recurso especies de árbol-nido $(B \mathrm{st}=0.246)$.

Características de los árboles-nido: El diámetro promedio a la altura del pecho de los árboles-nido fue de $72.4 \pm 39.5 \mathrm{~cm}$, y presentaron una altura promedio de la cavidad de 14.7 $\pm 4.2 \mathrm{~m}$. La mayoría de los nidos se encontraron en árboles con $\mathrm{DAP} \geq 60 \mathrm{~cm}(45.7 \%$, $\mathrm{n}=42$ ), el $38 \%$ se encontraron en árboles con DAP de 41 a $59 \mathrm{~cm}(\mathrm{n}=35)$, y sólo $16.3 \%$ $(\mathrm{n}=15)$ de los árboles tuvieron $\mathrm{DAP} \leq 40 \mathrm{~cm}$. La especie de árbol-nido con mayor dimensión fue Enterolobium cyclocarpum con una media de DAP mayor a $100 \mathrm{~cm}$ (Cuadro 1). Comparando las dimensiones (DAP y la altura de la cavidad) de los árboles-nido de este estudio, con los registrados en Tamaulipas, se encontraron diferencias significativas en el DAP $(U=6.19$, $\mathrm{p}=0.012)$, y en la altura de la cavidad $(U=31.6$, $\mathrm{p}<0.001)$. Los árboles-nido de Tamaulipas presentaron un DAP mayor $(78.8 \pm 41.5)$, pero una altura menor de la entrada de la cavidad

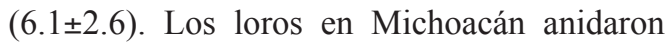
en cavidades ubicadas $\geq 10 \mathrm{~m}$ del suelo $(88.9 \%$, $\mathrm{n}=80$ ), con la mayoría de cavidades entre 10 y $20 \mathrm{~m}$ del suelo $(83 \%, \mathrm{n}=75)$. Los árbolesnido presentaron en promedio 1.5 cavidades (DS=0.8), con un intervalo de 2 a 4 cavidades utilizables, y no se observó el uso simultáneo de distintas cavidades por diferentes parejas de loros.

Ubicación de los árboles-nido: Los árboles-nido se encontraron en el intervalo altitudinal entre 5 y $675 \mathrm{msnm}$ (Media $=234 \mathrm{msnm}$, $\mathrm{DS}=117.1 \mathrm{msnm})$, pero la mayoría $(55.4 \%)$ se registró entre 101 y 300msnm. La pendiente promedio de su ubicación fue de $15^{\circ}$ $\left(\mathrm{DS}=8.7^{\circ}\right)$, con el $35 \%$ de los nidos situados en pendiente ligera o nivelado $\left(\leq 10^{\circ}\right)$, pero $41 \%$ de los nidos estuvieron en terrenos inclinados $\left(11^{\circ}-20^{\circ}\right)$, y $24 \%$ de los nidos en terreno muy inclinado $\left(>20^{\circ}\right)$. Los árboles-nido no se localizaron en orientaciones de ladera específicas, sin embargo, se notó una tendencia de agregación hacia el Sureste, Sur, y Suroeste, con el $47 \%$ de los árboles-nido en esas orientaciones. En términos de posición topográfica, los nidos se encontraron principalmente en valles y 
laderas poco pronunciadas $(70.7 \%)$, y en menor proporción en faldas y pendientes pronunciadas $(21.7 \%)$, en crestas $(4.4 \%)$, y en laderas superiores $(3.3 \%)$.

Los árboles-nido se encontraron a una distancia promedio de $1439.4 \mathrm{~m}(\mathrm{DS}=995.3 \mathrm{~m})$ de ranchos o poblados (rango entre $132 \mathrm{~m}$ y 4 $039 \mathrm{~m}$ ). Sin embargo, el $62 \%$ de los nidos se ubicaron a más de $1 \mathrm{~km}$ de distancia de asentamientos humanos. Con respecto a caminos y carreteras, los nidos se localizaron a una distancia promedio de $6167 \mathrm{~m}$ (DS=4 453.5m), en un intervalo entre $10.4 \mathrm{~m}$ y $14965.5 \mathrm{~m}$, aunque el $82.6 \%$ de los nidos se encontraba a más de $2 \mathrm{~km}$ de caminos. Por el contrario, los nidos se encontraron en hábitats ribereños o a corta distancia de ellos, su ubicación en promedio fue de $49.9 \mathrm{~m}$ ( $\mathrm{DS}=56.9 \mathrm{~m})$, estando el nido más lejano a $230.3 \mathrm{~m}$. El $76.1 \%$ de los nidos estaban ubicados a menos de $50 \mathrm{~m}$ de hábitats ribereños.

Estructura de la comunidad arbórea alrededor de los árboles-nido: En 16 parcelas de $25 \mathrm{~m}$ de radio centradas en los nidos de tres áreas con distintas condiciones de manejo, se midieron e identificaron 426 árboles (DAP $\geq 10 \mathrm{~cm})$ pertenecientes a 27 familias y 54 especies (Apéndice). En general, la estructura arbórea donde anidaron los loros presentó tres estratos: un estrato alto con pocos árboles emergentes, siendo frecuentemente el árbolnido el de mayor tamaño $(\mathrm{n}=18,4 \%$, o 34 , incluyendo los árboles-nido) que varió de 17 a $23 \mathrm{~m}$, un estrato intermedio con árboles que midieron desde 10 hasta $16 \mathrm{~m}(\mathrm{n}=151 ; 35 \%)$, y un estrato arbóreo de menor altura $(n=203$, $48 \%$ ), con árboles de 3 a $9 \mathrm{~m}$.

La riqueza promedio de especies arbóreas observada alrededor de los nidos fue de 14.3 especies, existiendo diferencias entre las tres áreas. La menor riqueza se observó alrededor de los nidos $(\mathrm{n}=3)$ del rancho "El Habillo", área con intensa actividad ganadera, cuyo promedio fue de 12.3 especies (intervalo 10-14). En contraste, la mayor riqueza promedio fue de 15 especies (intervalo 13-22) alrededor de los nidos $(\mathrm{n}=8)$ del rancho "La Bonetera", área que está en regeneración natural. Mientras que en el área de extracción comunal "Motín del Oro" se registró un promedio de 14.6 especies (intervalo 12-17). A nivel área, el menor número de familias (17) y especies (21) se registró en "El Habillo", y el máximo con 24 familias y 41 especies se observó en "La Bonetera". Mientras que en Motín del Oro se observó una riqueza intermedia con 21 familias y 28 especies.

La mayoría de los árboles (389) se identificaron a nivel especie o género, 13 árboles se identificaron a nivel de familia, y 24 no pudieron ser identificados. Las familias con el mayor número de especies fueron: Fabaceae (6), Burseraceae (4) y Caesalpiniaceae (4). Con base en las observaciones de campo, entrevistas y lo registrado en literatura, 27 especies $(50 \%)$ son utilizadas como alimento (Apéndice). Las familias con mayor número de especies que aportan recursos alimenticios a los loros cabeza-amarilla son Burseraceae (Bursera simaruba, Bursera instabilis, Bursera excelsa y Bursera sp.), Mimosaceae (Enterolobium cyclocarpum, Lysiloma microphyllum y Pithecellobium dulce), seguidas de Anacardiaceae, Bombacaceae, Boraginaceae y Moraceae, cada familia con dos especies (Apéndice).

Con respecto a las áreas estudiadas, el menor número de familias $(\mathrm{n}=11)$ y especies $(n=13)$ con uso potencial en la dieta del loro cabeza-amarilla se registró en "El Habillo", y el mayor número de familias ( $\mathrm{n}=15)$ y especies $(\mathrm{n}=19)$ se observó en "La Bonetera". En Motín del Oro se identificaron 14 familias y 15 especies que proporcionan alimento al loro.

$\mathrm{Al}$ analizar en conjunto todos los árboles muestreados de las tres áreas, 228 (53\%) árboles son de especies que utiliza el loro cabezaamarilla en su dieta. Las especies de árboles con mayor abundancia y densidad estimada fueron: Astronium graveolens $(\mathrm{n}=41 ; 10.1-25.4$ / ha), Brosimum alicastrum ( $\mathrm{n}=34 ; 10.1-56.0 / \mathrm{ha})$, Bursera simaruba ( $\mathrm{n}=20 ; 5.0-20.3 / \mathrm{ha})$, y Lysiloma microphyllum $(\mathrm{n}=18 ; 10.1-20.3 / \mathrm{ha})$, todas proporcionan alimento al loro cabeza-amarilla, y dos proporcionan además sitios de anidación.

También se observó alimentación en árboles fuera de los sitios de anidación pero cercanos $(\leq 500 \mathrm{~m})$, incluyendo: Astianthus viminalis 
(Bignoniaceae), Mangifera indica (Anacardiaceae) y Enterolobium cyclocarpum (Mimosaceae) (Fig. 2).

Proporciones de presencia de nidos por vegetación: Los árboles-nido se encontraron en tres tipos de vegetación (bosque tropical caducifolio, bosque tropical subcaducifolio, bosque tropical subcaducifolio ribereño), divididos en seis categorías (Cuadro 1). El mayor número de nidos se registró en bosque tropical subcaducifolio ribereño (29.3\%), seguido de bosque tropical subcaducifolio conservado $(26 \%)$, y bosque tropical subcaducifolio en pequeñas arboledas $(\leq 0.5 \mathrm{ha})$ formando ecotonos con el bosque tropical caducifolio (22.8\%). El menor número de árboles-nido se localizó en el bosque tropical caducifolio perturbado $(4.3 \%$, $n=4$ ) (Cuadro 1). Al analizarse la proporción de presencia de 43 árboles-nido con base en la disponibilidad de los tipos de vegetación de cinco áreas muestreadas intensivamente (Chuquiapan $\mathrm{n}=6$; Arteaga $\mathrm{n}=15$; Motín del Oro $\mathrm{n}=10$; El Habillo $n=3$; y La Bonetera $n=9$ ) se observó que la proporción de presencia de nidos difiere significativamente de lo esperado con base en la disponibilidad de vegetación muestreada $\left(\mathrm{G}_{5}=40.1, \mathrm{p}<0.001\right)$. Se encontraron más nidos en el bosques tropical subcaducifolio conservado (incluyendo arboledas de ecotonos) que lo esperado con base en su disponibilidad. Por el contrario, se encontró una proporción menor de

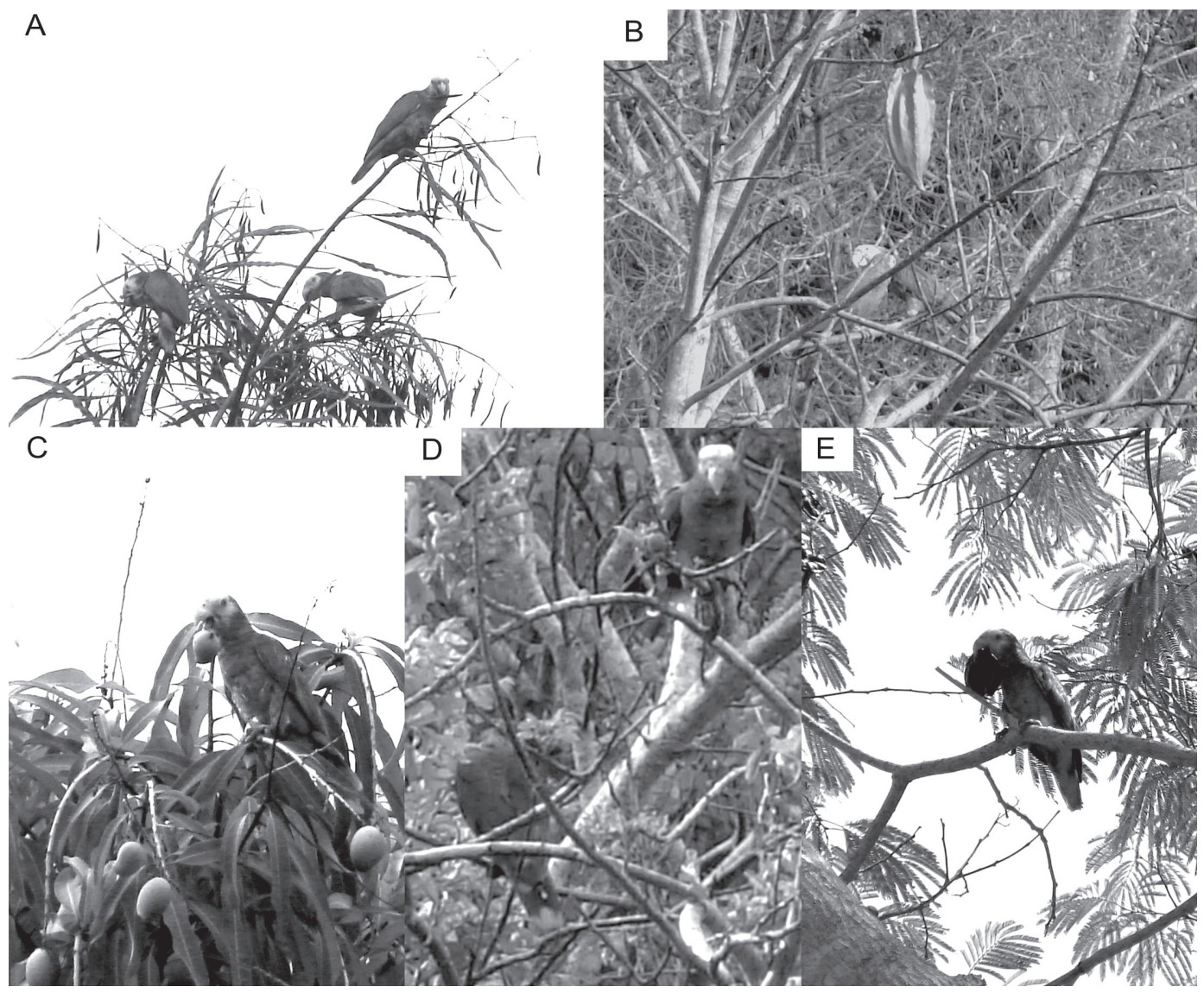

Fig. 2. Observaciones de alimentación de loro cabeza-amarilla en árboles nativos e introducidos. Astianthus viminalis (A), Jacaratia mexicana (B), Mangifera indica (C), Spondias purpurea (D) y Enterolobium cyclocarpum (E).

Fig. 2. Foraging observations of Yellow-headed Parrot using native and introduced trees. Astianthus viminalis (A), Jacaratia mexicana (B), Mangifera indica (C), Spondias purpurea (D) y Enterolobium cyclocarpum (E). 
nidos que los esperados, en el bosque tropical caducifolio conservado, y no se encontraron nidos en áreas agropecuarias (Cuadro 2).

Además de las diferencias en las proporciones de nidos entre tipos de vegetación, se observaron diferencias en algunas dimensiones entre nidos de los distintos tipos de vegetación. El diámetro de árboles-nido de arboledas presentaron un DAP menor ( $\mathrm{DAP}=49.5$, $\mathrm{DS}=19.4 \mathrm{~cm})$, que fue estadísticamente significativo $\left(\mathrm{H}_{3}=8.1, \mathrm{p}=0.04\right)$, al compararse con los árboles-nido encontrados en áreas de bosque tropical subcaducifolio continuo ( $\mathrm{DAP}=85.1$, $\mathrm{DS}=58.2 \mathrm{~cm}$ ). Sin embargo, no hubo diferencias significativas con respecto a la altura de la cavidad $\left(\mathrm{H}_{3}=0.62, \mathrm{p}=0.89\right)$.

Superficie con aptitud climática para anidación: Con base en el modelo de Maxent, las variables que se relacionan mayormente con la distribución de los nidos fueron: temperatura promedio del trimestre más lluvioso con $35.1 \%\left(\right.$ Media $=27.2^{\circ} \mathrm{C}$, rango $=25.1-28.9^{\circ} \mathrm{C}$ ), oscilación diurna de la temperatura con $22.3 \%$ (Media $=12.8^{\circ} \mathrm{C}$, rango $=12-14.8^{\circ} \mathrm{C}$ ), y precipitación del mes más lluvioso con 19.8\% (Media $=251 \mathrm{~mm}$, rango=140-306mm). En la región, la extensión que presenta condiciones de aptitud climática para la presencia potencial de nidos fue de $3432 \mathrm{~km}^{2}$, representando 40.4\% del área de estudio (Fig. 1). En el área climáticamente apta, los principales tipos de vegetación y su extensión son: el bosque tropical caducifolio con $1916 \mathrm{~km}^{2}$ (55.8\%), seguida de la superficie agropecuaria con $684 \mathrm{~km}^{2}$ (19.9\%), el bosque tropical subcaducifolio que incluye los bosques de ribera con $587 \mathrm{~km}^{2}$ (17\%), los bosques templados de pino-encino que ocupan $215 \mathrm{~km}^{2}(6.2 \%)$, y la superficie restante $(\leq 1.1 \%)$ corresponde a cuerpos de agua y asentamientos humanos.

\section{DISCUSIÓN}

En el Pacífico, el loro cabeza-amarilla presenta condiciones adversas para su reproducción, ya que los resultados mostraron que la especie requiere de condiciones muy específicas para anidar, tanto a escala local (especies de árbol-nido y características, ubicación, estructura del bosque, y dieta) como a nivel paisaje (localización con respecto a caminos y poblados, disponibilidad de tipos de vegetación, tolerancia a distintos tipos de manejo, área con aptitud climática), la suma de requerimientos tan específicos permiten entender cómo la degradación y destrucción de los bosques tropicales ha influido en su distribución en la región (Monterrubio-Rico et al., 2007).

En general, el loro cabeza-amarilla seleccionó los árboles-nido en forma similar a otros psitácidos, utilizó árboles con diámetros

CUADRO 2

Intervalos de confianza de Bonferroni comparando la proporción de presencia observada de 43 nidos con la proporción esperada para cada tipo de vegetación en cinco áreas muestreadas intensivamente

TABLE 2

Bonferroni confidence intervals comparing the presence proportion of 43 nests to the expected proportion by vegetation types in five areas surveyed intensively

\begin{tabular}{lcccc}
\multicolumn{1}{c}{ Tipo de vegetación } & Área (has) & $\begin{array}{c}\text { Proporción } \\
\text { esperada }\end{array}$ & $\begin{array}{c}\text { Proporción } \\
\text { observada }\end{array}$ & $\begin{array}{c}\text { Intervalos } \\
\text { de Bonferroni }\end{array}$ \\
Bosque tropical subcaducifolio conservado** & 171.3 & 0.038 & 0.51 & $0.313 \leq$ obs $\leq 0.706^{*}$ \\
Bosque tropical subcaducifolio perturbado & 852 & 0.191 & 0.20 & $0.042 \leq$ obs $\leq 242$ \\
Bosque tropical subcaducifolio ribereño & 312 & 0.07 & 0.12 & $0 \leq$ obs $\leq 0.247$ \\
Bosque tropical caducifolio conservado & 2371 & 0.53 & 0.09 & $0 \leq$ obs $\leq 0.202^{*}$ \\
Bosque tropical caducifolio perturbado & 378.0 & 0.084 & 0.046 & $0 \leq$ obs $\leq 0.128$ \\
Áreas agropecuarias & 367.7 & 0.08 & 0 & - \\
\hline
\end{tabular}

$* \mathrm{p}<0.05 * *$ Incluye arboledas $(\leq 0.5 \mathrm{ha})$ de bosque tropical subcaducifolio en bosque tropical caducifolio conservado. 
amplios $(\mathrm{DAP} \geq 60 \mathrm{~cm})$ y cavidades a la altura de dosel, concentrando sus nidos en pocas especies de árboles o árboles clave con base en la disponibilidad local de árboles (Keystone trees) (Marsden \& Jones, 1997; Renton \& Salinas-Melgoza, 1999; Brightsmith, 2005; Rodríguez-Castillo \& Eberhard, 2006; Monterrubio-Rico, Ortega-Rodríguez, Marín-Togo, Salinas-Melgoza \& Renton, 2009). Aunque el loro cabeza-amarilla fue observado usando cavidades en 11 distintas especies de árboles, concentró el $72.8 \%$ de sus nidos en sólo dos especies de árboles (Astronium graveolens y Enterolobium cyclocarpum), y este porcentaje de concentración de nidos en el menor número de especies de árboles, es de los más altos registrado para psitácidos en Mesoamérica. Esta concentración de nidos en pocas especies puede evidenciar una baja disponibilidad de árboles adecuados para anidar en la región, ya que una condición similar se observó en el loro corona-lila (Amazona finschi) con $62 \%$ de los nidos en las mismas especies de árboles en la misma región (Monterrubio-Rico et al., 2009). Sin embargo, el uso de pocas especies de árboles también se ha observado en otras regiones de Mesoamérica, en distintas condiciones y tipos de vegetación. Por ejemplo, en Panamá el loro corona amarilla (Amazona ochrocephala) concentró $63 \%$ de los nidos en dos especies (Roystonea regia y Rhizophora mangle), aunque utilizó ocho especies de árboles para anidar (Rodríguez-Castillo \& Eberhard, 2006). En Costa Rica, la guacamaya escarlata (Ara macao) concentró el 59\% de sus nidos en dos especies (Schizolobium parahybum y Ceiba pentandra), aunque anidó en 11 especies de árboles (Vaughan, Nemeth, \& Marineros, 2003). Sin embargo, la concentración de nidos observada en el loro cabeza-amarilla en este estudio es elevada, incrementando su vulnerabilidad ante plagas, enfermedades y tala selectiva que pudiesen padecer Astronium graveolens y Enterolobium cyclocarpum. Desafortunadamente, la tala selectiva no reglamentada es común en la región, donde las especies maderables de cualquier tipo de bosque son taladas en cuanto alcanzan dimensiones comerciales (30 a 40cm de DAP). Este tipo de manejo forestal crea fragmentos de bosque con árboles altos pero con una estructura de diámetros delgados y uniformes, y baja disponibilidad de cavidades adecuadas para que aniden loros y otras aves (Marsden \& Pilgrim, 2003; Cockle, Martin, $\&$ Wiebe, 2008). El elevado nivel de concentración de nidos en las mismas especies de árboles por parte de los loros cabeza-amarilla y corona-lila en el Pacífico central en Michoacán, posiblemente sean evidencia de la baja disponibilidad de cavidades en el bosque tropical subcaducifolio, o la poca disponibilidad de bosque tropical subcaducifolio de la región, por lo que $A$. graveolens y E. cyclocarpum e incluso Brosimum alicastrum deben ser considerados especies clave para ambos loros, y su tala debe ser suspendida, promoviendo su uso en la reforestación para la región.

Aunque los nidos del loro cabeza-amarilla se encontraron en árboles de diámetros $\geq 60 \mathrm{~cm}$, similares a los registrados en el Golfo de México (Enkerlin-Hoeflich, 2005), se observaron diferencias en la altura de la cavidad, siendo la altura promedio observada en el Pacífico de casi el doble de la altura promedio reportada en el Golfo de México. Esta diferencia se puede explicar parcialmente por dos causas: diferente disponibilidad de cavidades a mayor altura entre los distintos tipos de vegetación presentes en ambas vertientes, y diferencias de presión de saqueo de cada área que influyen en la tolerancia del loro para anidar a menor altura. Ante el fuerte saqueo de nidos en el Pacífico, es posible que la respuesta conductual del loro cabezaamarilla sea similar a la que tendría ante una fuerte depredación, seleccionando cavidades a las mayores alturas posibles, ocultando sus nidos en el follaje del dosel del bosque tropical subcaducifolio (Monterrubio-Rico et al., 2007). En psitácidos se ha observado que los nidos en cavidades a una mayor altura presentan mayor supervivencia y menor depredación (Berkunsky \& Reboreda, 2009). Por el contrario, ante la ausencia de saqueo de nidos en el estudio efectuado en el Golfo de México, el loro cabeza-amarilla anidó en cavidades a menor altura, a pesar de que en general, las aves padecen 
mayor depredación a menor altura (Nilsson, 1984; Wilcove, 1985; Marsden \& Jones, 1997). Los próximos estudios deben evaluar la relación entre la disponibilidad y uso por los loros de cavidades en cada tipo de vegetación a distintas alturas, en presencia y ausencia de saqueo de nidos, y paralelamente evaluar los niveles de depredación, para probar estas hipótesis y diseñar mejores medidas de manejo.

Aunque los árboles-nido se encontraron en un intervalo de elevación amplio (5-675 msnm), este es ligeramente inferior al límite superior reportado para su distribución general $(0$ a 900msnsm) (Howell \& Webb, 1995), además, de que las áreas de anidación se ubicaron principalmente en la región costa, y aunque se registraron algunos nidos en la Sierra Madre del Sur, estos se encontraron a bajas elevaciones en barrancas con vegetación tropical. El hecho de que la mayoría de los árboles-nido se ubicaron entre 101 y 300msnm, está influido en parte por la disponibilidad del bosque tropical subcaducifolio a esa elevación, ya que las planicies costeras a menor elevación $(<100 \mathrm{msnm})$, están dominadas por paisajes agropecuarios, en forma similar a lo previamente observado para el loro corona-lila (Amazona fisnchi) (OrtegaRodríguez \& Monterrubio-Rico, 2008).

Otro requerimiento en la anidación del loro cabeza-amarilla parece ser el anidar lejos de asentamientos humanos, los cuales en su mayoría ocurren también a baja elevación y a lo largo de la carretera costera del Pacífico, pues el $62 \%$ de los árboles-nido se ubicó a más de $1 \mathrm{~km}$ de poblados, y más del $80 \%$ de los nidos se encontraron a más de $2 \mathrm{~km}$ de carreteras y caminos principales. Posiblemente, también se relacione con la menor disponibilidad de hábitat adecuado para anidar cerca a los asentamientos humanos y áreas agropecuarias, donde la extracción de árboles es facilitada por la presencia de caminos. Así mismo la presión de saqueo puede ser mayor, debido al fácil acceso, en los bosques cercanos a asentamientos humanos y carreteras. Por otra parte, parecen estar asociados a arroyos y ríos, ya que más del $75 \%$ de los nidos se ubicaron a menos de $50 \mathrm{~m}$ de ríos o arroyos, que se explica en parte por una mayor disponibilidad de árboles de mayor tamaño cerca del agua, y en terrenos relativamente menos accesibles para la tala.

El loro cabeza-amarilla parece anidar en bosques tropicales subcaducifolios de las áreas menos accesibles, y distantes a poblados y caminos, es decir en las áreas de bosque en mejor condiciones de conservación, y así se observó para una gran mayoría de sus nidos. Sin embargo los bosques de la región entera parecen haber sido ya manejados en mayor o menor grado. Aunque en los fragmentos de bosque tropical donde anidó el loro se observó una riqueza importante de especies de árboles que le proporcionan alimento, esta riqueza mostró variación asociada a las condiciones de manejo. Al comparar propiedades con diferentes condiciones de manejo se observó un mayor número de nidos y especies de árboles en el rancho con bosque tropical subcaducifolio en regeneración natural mientras que el menor número de nidos activos y especies que proporcionan alimento se observó en el rancho con bosque tropical muy fragmentado e intensas actividades ganaderas en desarrollo (El Habillo).

Una característica que presentaron los fragmentos de bosque donde anidó el loro cabeza-amarilla fue que en las especies más abundantes (Astronium graveolens, Bursera simaruba, Lysiloma divaricatum y Brosimum alicastrum), sus semillas y frutos proporcionan alimento a los loros. Además, en general, existe similitud entre las especies que forman parte de la dieta del loro cabeza-amarilla y otros psitácidos en Mesoamérica. En los bosques del oeste de Costa Rica (Matuzak, Bezy \& Brightsmith, 2008), el loro nuca-amarilla (Amazona auropalliata), y el loro frente-blanca (Amazona albifrons) se alimentaron de las mismas especies o de especies del mismo género. Es posible que las distintas comunidades de loros en el neotrópico presenten requerimientos nutricionales similares, y los satisfagan en las mismas especies de árboles o especies similares, evidenciando la estrecha relación que parece existir en las agregaciones (assemblages) de especies en el trópico de Mesoamérica. 
Aunque el grado en que la disponibilidad de recursos alimenticios en un fragmento de bosque influye en la selección del sitio de anidación, este es poco conocido para la mayoría de los psitácidos neotropicales. Nuestros resultados parecen indicar que existe un conjunto de características que influyen en la selección del área en el bosque para anidar. Hipotetizamos que además de las dimensiones de cavidades es importante la presencia de riqueza y abundancia específica de árboles que proporcionan alimento, tanto en la vecindad de los nidos, como a corta distancia. En este sentido, es necesario diseñar adecuadamente un estudio con el fin de comprender mejor cómo la riqueza y abundancia de árboles dieta se relaciona con los árboles-nido y la relación entre el tamaño y el área de actividad de las parvadas de alimentación con la disponibilidad de alimento. Esta información es relevante para el diseño de estrategias de conservación y restauración del paisaje fragmentado de la costa central del Pacífico, donde además la vegetación presenta variación estacional en la disponibilidad de recursos.

Por otra parte, las especies de árboles Astronium graveolens, Brosimum alicastrum, Bursera simaruba, Cordia alliodora y Enterolobium cyclocarpum deben ser consideradas especies clave para la supervivencia de los loros de la región, ya que además de proveer cavidades, también proporcionan alimento a las dos especies de loros en peligro de extinción en el Pacífico central mexicano (Renton, 2001; Monterrubio-Rico et al., 2009).

Además de los requerimientos a nivel local, la extensión de los diferentes tipos de vegetación explican los niveles de abundancia de nidos en distintas áreas de la región. Por ejemplo, aunque el bosque tropical caducifolio es el tipo de vegetación con mayor extensión, fue utilizado por los loros en una proporción significativamente menor de lo esperado, a pesar de que en el Pacífico el mayor porcentaje de parejas y parvadas de loros en vuelo y perchados, han sido registrados en este tipo de vegetación (Monterrubio-Rico et al., 2010). En contraste, el bosque tropical subcaducifolio fue usado para anidar más de lo esperado con base en su extensión. Se explica a partir de que este tipo de bosque presenta las especies de árboles con las características estructurales para la formación de cavidades a la altura de dosel, encontrándose la mayoría de nidos en Astronium graveolens, Enterolobium cyclocarpum y Brosimum alicastrum, especies que forman parte del dosel tanto del bosque tropical subcaducifolio, como de áreas de ecotonos con bosque tropical caducifolio.

Desafortunadamente, el bosque tropical subcaducifolio en la región presenta una baja cobertura, ya no existen grandes fragmentos continuos, y los remanentes consisten en arboledas $(\leq 1 \mathrm{ha})$ en ecotonos, y los árboles-nido de las arboledas presentaron un menor DAP. El menor grosor de los árboles al nivel de la cavidad podría reducir la productividad del loro, ya que se sabe que el uso de cavidades de menor diámetro influye en el tamaño de nidada en otras aves (Wiebe, Koenig, \& Martin, 2001). En el Pacifico central, las arboledas de bosque tropical subcaducifolio se presentan en ecotonos con el bosque tropical caducifolio, asociadas a manantiales o laderas con mayor humedad, presentando en su composición árboles del bosque tropical subcaducifolio. La anidación en árboles-nido de menor diámetro en arboledas podría deberse a la disminución del bosque tropical subcaducifolio maduro durante las últimas décadas en la región, reduciéndose la disponibilidad de cavidades en árboles grandes a nivel regional. Es importante evaluar pronto la disponibilidad y el diámetro interno de las cavidades, así como el desempeño reproductivo entre los diferentes hábitats disponibles, para diseñar y aplicar acciones de restauración en la región, antes de que aumente el cambio en el uso del suelo.

En general, pocos estudios sobre loros Amazona han documentado una mayor abundancia de nidos en áreas manejadas y agropecuarias que en el bosque natural o nativo para anidar, como es el caso de Amazona aestiva en el pantanal de Brasil (Seixas \& Mourão, 2002). En el caso del loro cabeza-amarilla parecería que existen evidencias contrastantes, ya que en el Golfo de México, los árboles-nido 
estudiados en los ochentas se localizaron en áreas manejadas con arboledas y pastizales de un rancho ganadero (Enkerlin-Hoeflich, 1995), mientras que en el Pacífico el rancho ganadero estudiado presentó el menor número de nidos. Las diferencias contrastantes entre el Golfo de México y el Pacífico puede explicarse por varias razones. El tamaño de muestra en el Golfo de México fue pequeño, limitado a los nidos de un rancho ganadero, mientras que en el Pacífico se estudió al loro en diferentes áreas y condiciones. A escala local, los resultados reflejan las diferencias en disponibilidad de cavidades adecuadas entre los tipos de vegetación presentes, y posiblemente las diferencias de presión de saqueo de cada área. Por lo que una menor presión de saqueo explicaría la selección de sitios más abiertos y expuestos en el Golfo de México.

Aunque la región del Pacifico central que se estudió es extensa, con más de $6000 \mathrm{~km}^{2}$, las condiciones de aptitud climática para la presencia potencial de nidos de loro cabezaamarilla sólo se presentan en el $61.2 \%$ de la región, y $27.2 \%$ de esa superficie corresponde a zonas agropecuarias, o bosques templados de pino-encino, cuerpos de agua y asentamientos humanos donde no anidan los loros, mientras que la vegetación tropical que podría usar el loro para anidar corresponde sólo al $72.8 \%$ del área con aptitud climática para anidar. Aunque existen estimaciones de distribución potencial para la especie tanto a nivel regional como para todo el Pacífico (Monterrubio-Rico et al., 2007; Monterrubio-Rico et al., 2010), estas estimaciones no son útiles para cuantificar la extensión de áreas de anidación para la especie, ya que las áreas de aptitud climática para la anidación potencial son mucho menores a las estimaciones de distribución potencial, además de que existen diferencias sustanciales en la abundancia de nidos entre tipos de vegetación, por lo que cuantificar el hábitat de anidación disponible para cada población reproductora debe ser una prioridad para conservar la especie, y en este estudio se proporciona la primera estimación de este tipo.
Desafortunadamente, los bosques tropicales estacionales del Pacífico central están siendo transformados rápidamente en las áreas de distribución del loro cabeza-amarilla dentro de México (Trejo \& Dirzo, 2000; Miles et al., 2006), por lo que urge delimitar y estimar las áreas de aptitud para su anidación en cada área remanente. Ante la falta de una metodología clara para generar modelos predictivos de áreas de aptitud para anidación, recomendamos procedimientos metodológicos que incluyan la estimación de áreas climáticamente aptas para la presencia de nidos a partir de una muestra representativa de nidos localizados en los distintos tipos de vegetación de la región, delimitando los tipos de vegetación importantes para su anidación dentro de dicha área.

Varios requerimientos para anidar del loro cabeza-amarilla hacen que la especie sea muy vulnerable en el Pacífico central, destacamos su anidación casi exclusiva en bosque tropical subcaducifolio, siendo el tipo de bosque que presenta la menor extensión y ocupa sólo el $17 \%$ del área en condiciones de aptitud ambiental para la presencia potencial de nidos en la región. Este tipo de vegetación también fue documentado como el más importante para el loro corona-lila, y se reporta como clave para varias especies de vertebrados, especialmente durante la estación seca ya que proporciona alimento y refugio (Renton, 2001; MonterrubioRico et al., 2009).

La anidación preferencial del loro en el bosque tropical subcaducifolio se entiende al ser el tipo de bosque con la mayor densidad de árboles grandes (Lott, Bullock, \& Solís-Magallanes, 1987), además destaca la importancia de mantener toda la extensión actual de este tipo de vegetación dentro de la zona de aptitud climática para anidar, delimitada en este estudio. Por lo tanto, se debe prohibir el cambio de uso de suelo en este tipo de vegetación. Ante la falta de áreas naturales protegidas en el Pacífico central en la costa de Michoacán, y ante la falta de vigilancia para aplicar la prohibición de saqueo de nidos, es necesario maximizar la disponibilidad de bosque tropical subcaducifolio, promoviendo su restauración y estableciendo 
áreas protegidas en los municipios de Arteaga y Aquila, para incrementar la representatividad de este tipo de bosque (Villaseñor-Gómez, Robles-del Valle, Briseño-Cázares, \& AmadorGarcía, 2005).

En la costa de Michoacán, algunas comunidades indígenas (Coire y Pómaro, municipio de Aquila) todavía mantienen grandes áreas de bosque tropical continuo, sin embargo en sus usos y costumbres capturan los pollos de los loros para el mercado regional y nacional de mascotas. Por lo que urge un acuerdo de colaboración entre las comunidades indígenas y las agencias gubernamentales para promover los programas de "pago por servicios ambientales" que protejan los árboles-nido conocidos, los árboles con cavidades, y los hábitats de anidación, además de prohibir en forma determinante la extracción de pollos. También deben establecerse acuerdos similares con las cooperativas de tierras comunitarias conocidas como "ejidos", y con pequeñas propiedades privadas donde existen áreas de anidación en bosque tropical subcaducifolio. Además, las acciones de conservación a largo plazo del loro cabezaamarilla proporcionarían nuevas actividades económicas recreativas para la región en ecoturismo de observación de aves, en particular de loros. Por lo tanto, se deben implementar estrategias regionales que mantengan los árboles con cavidades en todos los tipos de vegetación, ya que son esenciales para la conservación de comunidades de especies que anidan en ellas, y reduciría la posible competencia entre especies para anidar (Cornelius et al., 2008)

Este es el primer estudio que brinda la información suficiente para proteger por primera vez de forma efectiva en México una población reproductora y viable de loro cabezaamarilla claramente delimitada. La conservación a largo plazo de sus áreas de anidación beneficiarían tanto a las especies de loros en peligro de extinción (loros cabeza-amarilla y corona-lila), como a la comunidad de aves que anidan en cavidades. Ahora corresponde a autoridades de distintos niveles crear condiciones para lograrlo.

\section{AGRADECIMIENTOS}

Apreciamos los consejos de logística de Laura y Fernando Villaseñor-Gómez. La ayuda en campo fue proporcionada por Edna López-Córdova, Beneiza Fabián-Turja, Berenice Santiago-Valencia, Adriano Ortega, Ismael Carrillo-Acevedo, Miguel Ángel De LabraHernández, Juan Felipe Charre-Medellín y Mario Quetzal Pureco-Rivera. Agradecemos la ayuda y la asistencia de Xavier Madrigal y Gabriela Domínguez en la identificación botánica. Agradecemos a Consuelo Marín-Togo por su asesoría en el uso de los sistemas de información geográfica. La investigación fue financiada por la Coordinación de la Investigación Científica de la Universidad Michoacana de San Nicolás de Hidalgo (UMSNH), y por el Fondo Sectorial de Investigación Ambiental CONACyT-SEMARNAT. Agradecemos a la Dirección General de Vida Silvestre de la SEMARNAT por los permisos para llevar a cabo la investigación. La Facultad de Biología (UMSNH) proporcionó las instalaciones para redactar este manuscrito. Agradecemos los comentarios de los revisores anónimos que sin duda ayudaron a mejorar este trabajo.

\section{RESUMEN}

Los requerimientos de anidación del loro cabezaamarilla (Amazona oratrix) son poco conocidos a pesar de su amplia distribución histórica, la alta demanda como mascota y estar en peligro de extinción. Esta información es necesaria para diseñar acciones específicas de restauración y conservación. Durante diez años estudiamos la ecología de anidación del loro en Michoacán, México. Las variables estudiadas incluyeron en escala local las especies de árboles-nido, sus dimensiones y posición geográfica, dieta y estructura de parches de bosque; y a mayor escala el uso de la vegetación y las variables climáticas asociadas a la distribución de árboles-nido, obtenidas con un modelo de nicho ecológico utilizando Maxent. También evaluamos la tolerancia del loro al manejo de la tierra, y comparamos los árboles-nido encontrados, con 18 árbolesnido de Tamaulipas (Golfo de México), estudiados en los ochentas en un rancho privado intensamente manejado. Los loros anidaron en árboles altos en cavidades del dosel, registramos 92 árboles-nido en 11 especies de árboles, pero el $72.8 \%$ se presentó en Astronium graveolens y Enterolobium cyclocarpum que califican como árboles clave. Los bosques de anidación presentan 54 especies de árboles 
como máximo, y 50\% sirven como alimento, también las zonas presentan alta abundancia de árboles dieta. La menor riqueza de árboles y de árboles dieta se presentó en un rancho ganadero, mientras que las áreas en regeneración natural presentaron mayor riqueza. La altura de la entrada de la cavidad en los árboles-nido del Pacífico fue mayor que en los del Golfo de México. Hipotetizamos que las diferencias se deben al comportamiento del loro, adaptado a la presión diferencial de saqueo de nidos y disponibilidad de cavidades. Los árboles-nido se encontraron en seis tipos de vegetación, pero el loro prefirió el bosque tropical subcaducifolio conservado, y ribereño, anidó menos en bosque tropical caducifolio y nunca en campos agrícolas transformados, difiriendo de lo esperado por la disponibilidad de hábitat. Las principales variables climáticas asociadas con la distribución potencial de nidos son: temperatura promedio del trimestre más lluvioso, oscilación diurna de la temperatura, y precipitación del mes más lluvioso. Las áreas de presencia potencial de árboles-nido representan el $61 \%$ de la región, sin embargo la mayor parte es bosque tropical caducifolio (55.8\%), y el bosque tropical subcaducifolio sólo ocupa $17 \%$. Los resultados indican la importancia del bosque tropical subcaducifolio conservado como hábitat de reproducción del loro cabeza-amarilla, y muestran la urgente necesidad de implementar acciones de conservación y restauración. Las acciones deben incluir la prohibición del cambio de uso del suelo del bosque tropical subcaducifolio, y de la tala selectiva de especies clave, la creación de santuarios de vida silvestre en las áreas de anidación y programas de restauración de los bosques tropicales de la costa del Pacífico central.

Palabras clave: hábitat de anidación, composición de la dieta, condiciones climáticas.

\section{REFERENCIAS}

Berkunsky, I. \& Reboreda, J. C. (2009). Nest-site fidelity and cavity reoccupation by Blue-fronted Parrots Amazona aestiva in the dry Chaco of Argentina. Ibis, 151, 145-150.

Beyer, H. L. (2004). Hawth's Analysis Tools for ArcGIS (Version 3.x). Retireved from http://www.spatialecology.com/htools.

Brightsmith, D. J. (2005). Parrot nesting in Southeastern Peru: seasonal patterns and keystone trees. Wilson Bulletin, 117(3), 296-305.

Byers, C. R., Steinhorst, R. K., \& Krausman, P. R. (1984). Clarification of a technique for analysis of utilizationavailability data. Journal of Wildlife Management, 48(3), 1050-1053.

Cantú-Guzmán, J. C., Sánchez-Saldaña, M. E., Grosselet, M., \& Silva-Gámez, J. (2007). Tráfico ilegal de pericos en México: una evaluación detallada (p. 75). Washington, D.C.: Defenders of Wildlife.
Carranza-González, E. (2005). Vegetación. In L. E. Villaseñor-Gómez (Ed.), La biodiversidad en Michoacán: Estudio de Estado (pp. 38-45). México: Comisión Nacional para el Conocimiento y Uso de la Biodiversidad, Secretaría de Urbanismo y Medio Ambiente, Universidad Michoacana de San Nicolás de Hidalgo.

Cockle, K., Martin, K., \& Wiebe, K. (2008). Availability of cavities for nesting birds in the Atlantic forest, Argentina. Ornitología Neotropical, 19 (Suppl.), 269-278.

Collar, N. J., George, L. P., Krabbe, N., Madroño-Nieto, A., Naranjo, L. G., Parker III, T. A., \& Wege, D. C. (Eds.). (2000). Threatened birds of the Americas. Cambridge: Birdlife International.

Colwell, R. K. \& Futuyma, D. J. (1971). On the measurements of niche breadth and overlap. Ecology, 52(4), 567-576.

Cornelius, C., Cockle, K., Politi, N., Berkunsky, I., Sandoval, L., Ojeda, V., Rivera, L., Hunter Jr., M., \& Martin, K. (2008). Cavity-nesting birds in neotropical forests: cavities as a potentially limiting resource. Ornitología Neotropical, 19 (Suppl.), 253-268.

Delgado-Carrillo, O. (2012). Uso de recursos alimenticios por parte de aves en tres especies de árboles (Astronium graveolens, Spondias purpurea y Bursera simaruba) en bosques tropicales secos fragmentados y no fragmentados de la Costa de Michoacán (Tesis de Maestría). Universidad Michoacana de San Nicolás de Hidalgo, Morelia, Michoacán, México.

Enkerlin-Hoeflich, E. C. (1995). Comparative ecology and reproductive biology of three species of Amazona parrots in Northeastern Mexico (Tesis de Doctorado). Texas A \& M University, College Station, Texas, USA.

ESRI (Environmental Scientific Research Institute). (2008). ArcMap (Version 9.3). Redlands, California, USA: Environmental Scientific Research Institute.

Fabián-Turja, M. B. (2004). Abundancia relativa y características de anidación de loros del género Amazona en la costa de Michoacán (Tesis de Licenciatura). Universidad Michoacana de San Nicolás de Hidalgo, Morelia, Michoacán, México.

Forshaw, J. M. (1989). Parrots of the world (3rd ed.). Ontario: Silvio Mattacchione and Co.

Howell, S. N. G. \& Webb, S. (1995). A guide to the birds of Mexico, Northern and Central America. New York: Oxford University Press.

INEGI (Instituto Nacional de Estadística y Geografía). (2009). Carta de Uso del Suelo y Vegetación, Serie $I V$. Conjunto de datos vectoriales, México.

IUCN (International Union for Conservation of Nature). (2013). The IUCN Red List of Threatened Species (Version 2013.1.). Retrieved from http://www.iucnredlist.org 
Jenness, J., Brost, B., \& Beier, P. (2012). Land Facet Corridor Designer. USA. Retrieved from http://corridordesign.org/dl/tools/LandFacetCorridor.exe

Krebs, C. J. (1989). Ecological methodology. New York: Harper and Row.

Levins, R. (1968). Evolution in changing enviroment. New Jersey: Princeton University Press.

Lott, E. J., Bullock, S. H., \& Solís-Magallanes, J. A. (1987). Floristic diversity and structure of upland and arroyo forests of coastal Jalisco. Biotropica, 19(3), 228-235.

Marsden, S. J. \& Jones, M. J. (1997). The nesting requirements of the parrots and hornbill of Sumba, Indonesia. Biological Conservation, 82(3), 279-287.

Marsden, S. J. \& Pilgrim, J. D. (2003). Factors influencing the abundance of parrots and hornbills in pristine and disturbed forests on New Britain, PNG. Ibis, $145,45-53$.

Mas-Caussel, J. F., Velásquez-Montes, A., \& FernándezVargas, T. (2005). Monitoreo de los cambios de cobertura del suelo en Michoacán. In L. E. Villaseñor-Gómez (Ed.), La biodiversidad en Michoacán: Estudio de Estado (pp. 204-206). México: Comisión Nacional para el Conocimiento y Uso de la Biodiversidad, Secretaría de Urbanismo y Medio Ambiente, Universidad Michoacana de San Nicolás de Hidalgo.

Matuzak, G. D., Bezy, M. B., \& Brightsmith, D. J. (2008). Foraging ecology of parrots in a modified landscape: seasonal trends and introduced species. The Wilson Journal of Ornithology, 20(2), 353-365.

Miles, L., Newton, A. C., DeFries, R. S., Ravilious, C., May, I., Blyth, S., Kapos, V., \& Gordon, J. E. (2006). A global overview of the conservation status of tropical dry forests. Journal of Biogeography, 33, 491-505.

Monterrubio-Rico, T. C. \& Escalante-Pliego, P. (2006). Richness, distribution and conservation status of cavity nesting birds in Mexico. Biological Conservation, 128, 67-78.

Monterrubio-Rico, T. C., Ortega-Rodríguez, J. M., MarínTogo, M. C., Salinas-Melgoza, A., \& Renton, K. (2009). Nesting habitat of the Lilac-crowned Parrot in a modified landscape in Mexico. Biotropica, 41(3), 361-368.

Monterrubio-Rico, T. C., Renton, K., Ortega-Rodríguez, J. M., Pérez-Arteaga, A., \& Cancino-Murillo, R. (2010). The endangered yellow-headed parrot Amazona oratrix along the Pacific coast of Mexico. Oryx, 44(4), 602-609.

Monterrubio-Rico, T. C., Villaseñor-Gómez, L. E., MarínTogo, M. C., López-Córdova, E. A., Fabian-Turja, B., \& Sorani-Dalbon, V. (2007). Distribución histórica y actual del loro cabeza amarilla (Amazona oratrix) en la costa central del Pacífico mexicano: ventajas y limitaciones en el uso de GARP en especies bajo fuerte presión de tráfico. Ornitología Neotropical, 18, 263-276.

Morales-Pérez, L. (2005). Evaluación de la abundancia poblacional y recursos alimenticios para tres géneros de Psitácidos en hábitats conservados y perturbados de la costa de Jalisco, México (Tesis de Maestría). Universidad Nacional Autónoma de México, México, D. F.

Narayani, B. (2008). Tool for Partial-ROC (Version 1.0). Lawrence, Kansas, USA: Biodiversity Institute.

Neu, C. W., Byers, C. R., \& Peek, J. M. (1974). A technique for analysis of utilization-availability data. Journal of Wildlife Management, 38(3), 541-545.

Nilsson, S. G. (1984). The evolution of nest-site selection among hole-nesting birds: the importance of nest predation and competition. Ornis Scandinavica, 15, 167-175.

Ortega-Rodríguez, J. M. \& Monterrubio-Rico, T. C. (2008). Características geográficas de la ubicación de nidos del loro corona lila (Amazona finschi) en la Costa del pacífico en Michoacán, México. Ornitología Neotropical, 19, 427-439.

Pennington, T. D., \& Sarukhán, J. (2005). Árboles tropicales de México. Manual para la identificación de las principales especies. (3rd ed.). México, D. F.: UNAM/FCE.

Peterson, A. T., Papes, M., \& Soberón, J. (2008). Rethinking receiver operating characteristic analysis applications in ecological niche modeling. Ecological Modelling, 213, 63-72.

Phillips, S. J., Anderson, R. P., \& Schapire, R. E. (2006). Maximum entropy modeling of species geographic distributions. Ecological Modelling, 190, 231-259.

Phillips, S. J., Dudík, M., \& Schapire, R. E. (2004). A maximum entropy approach to species distribution modeling (pp. 655-662). Presented at the Proceedings of the $21^{\text {st }}$ International Conference on Machine Learning, New York, USA.: ACM Press.

Renton, K. (2001). Lilac-crowned parrot diet and food resource availability: resource tracking by a parrot seed predator. The Condor, 103, 62-69.

Renton, K. (2002). Amazona oratrix (Ridgway 1887) (Loro cabeza amarilla). In F. A. Noguera, J. H. Vega-Rivera, A. N. García-Aldrete, \& M. Quesada-Avendaño (Eds.), Historia Natural de Chamela (pp. 345-346). México D.F.: Universidad Nacional Autónoma de México.

Renton, K. \& Salinas-Melgoza, A. (1999). Nesting behavior of the Lilac-crowned Parrot. Wilson Bulletin, 111(4), 488-493.

Ridgely, R. S. (1981). The current distribution and status of mainland neotropical parrots. In R. S. Pasquier (Ed.), Conservation of New World parrots: Proceedings 
of the ICBP Parrot Working Group Meeting, St. Lucia, 1980. ICBP Technical Publication No. 1. (pp. 233-384). Washington, D.C.: Smithsonian Institution Press/ICBP.

Ríos-Muñoz, C. A. \& Navarro-Sigüenza, A. G. (2009). Efectos del cambio de uso de suelo en la disponibilidad hipotética de hábitat para los Psitácidos de México. Ornitología Neotropical, 20, 491-509.

Rodríguez-Castillo, A. M. \& Eberhard, J. R. (2006). Reproductive behavior of the Yellow-crowned Parrot (Amazona ochrocephala) in Western Panama. The Wilson Journal of Ornithology, 118(2), 225-236.

Rzedowski, J. (1994). Vegetación de México (6th ed.). México D. F.: Editorial Limusa.

SAS Institute Inc. (2001). SAS/STATTM. 2001. Guide for Personal Computers. (Version 8.2). North Carolina, USA: SAS Institute Inc.

Seixas, G. H. F. \& Mourão, G. de M. (2002). Nesting success and hatching survival of the Blue-fronted Amazon (Amazona aestiva) in the Pantanal of Mato Grosso do Sul, Brazil. Journal of Field Ornithology, 73(4), 399-409.

SEMARNAP-UNAM. (2000). Resultados del Inventario Forestal Nacional 2000. México, D.F.: Secretaría de Medio Ambiente, Recursos Naturales y Pesca, Instituto de Geología, Universidad Nacional Autónoma de México.

SEMARNAT. (2010). Norma Oficial Mexicana NOM059-SEMARNAT-2010, Protección ambiental-Especies nativas de México de flora y fauna silvestres-Categorias de riesgo y especificaciones para su inclusión, exclusión o cambio-Lista de especies en riesgo. México, DF.: Diario Oficial de la Federación. 30 de diciembre de 2010.

Snyder, N., McGowan, P., Gilardi, J., \& Grajal, A. (2000). Parrots status survey and conservation action plan
2000-2004. Gland and Cambridge: International Union for Conservation of Nature.

Stotz, D. F., Fitzpatrick, J. W., Parker III, T. A., \& Moskovits, D. K. (1996). Neotropical birds ecology and conservation. Chicago: University of Chicago Press.

Téllez-García, L. (2008). Abundancia relativa y características del hábitat de anidación del loro cabeza amarilla (Amazona oratrix) en diferentes condiciones de conservación de la vegetación (Tesis de Maestría). Universidad Michoacana de San Nicolás de Hidalgo, Michoacán, México.

Trejo, I. \& Dirzo, R. (2000). Deforestation of seasonally dry tropical forest: a national and local analysis in Mexico. Biological Conservation, 94, 133-142.

Vaughan, C., Nemeth, N., \& Marineros, L. (2003). Ecology and management of natural and artificial Scarlet Macaw (Ara macao) nest cavities in Costa Rica. Ornitología Neotropical, 14, 381-396.

Villaseñor-Gómez, L. E., Robles-del Valle, J. S., BriseñoCázares, L. A., \& Amador-García, A. (2005). Áreas naturales protegidas. In L. E. Villaseñor-Gómez (Ed.), La biodiversidad en Michoacán: Estudio de Estado (pp. 177-186). México: Comisión Nacional para el Conocimiento y Uso de la Biodiversidad, Secretaría de Urbanismo y Medio Ambiente, Universidad Michoacana de San Nicolás de Hidalgo.

Wiebe, K. L., Koenig, W. D., \& Martin, K. (2001). Evolution of clutch size in cavity-excavating birds: the nest site limitation hypothesis revisited. The American Naturalist, 167(3), 343-353.

Wilcove, D. S. (1985). Nest predation in forest tracts and the decline of migratory songbirds. Ecology, 66(4), 1211-1214.

Zar, J. H. (1996). Biostatistical analysis. New Jersey: Prentice Hall Inc. 


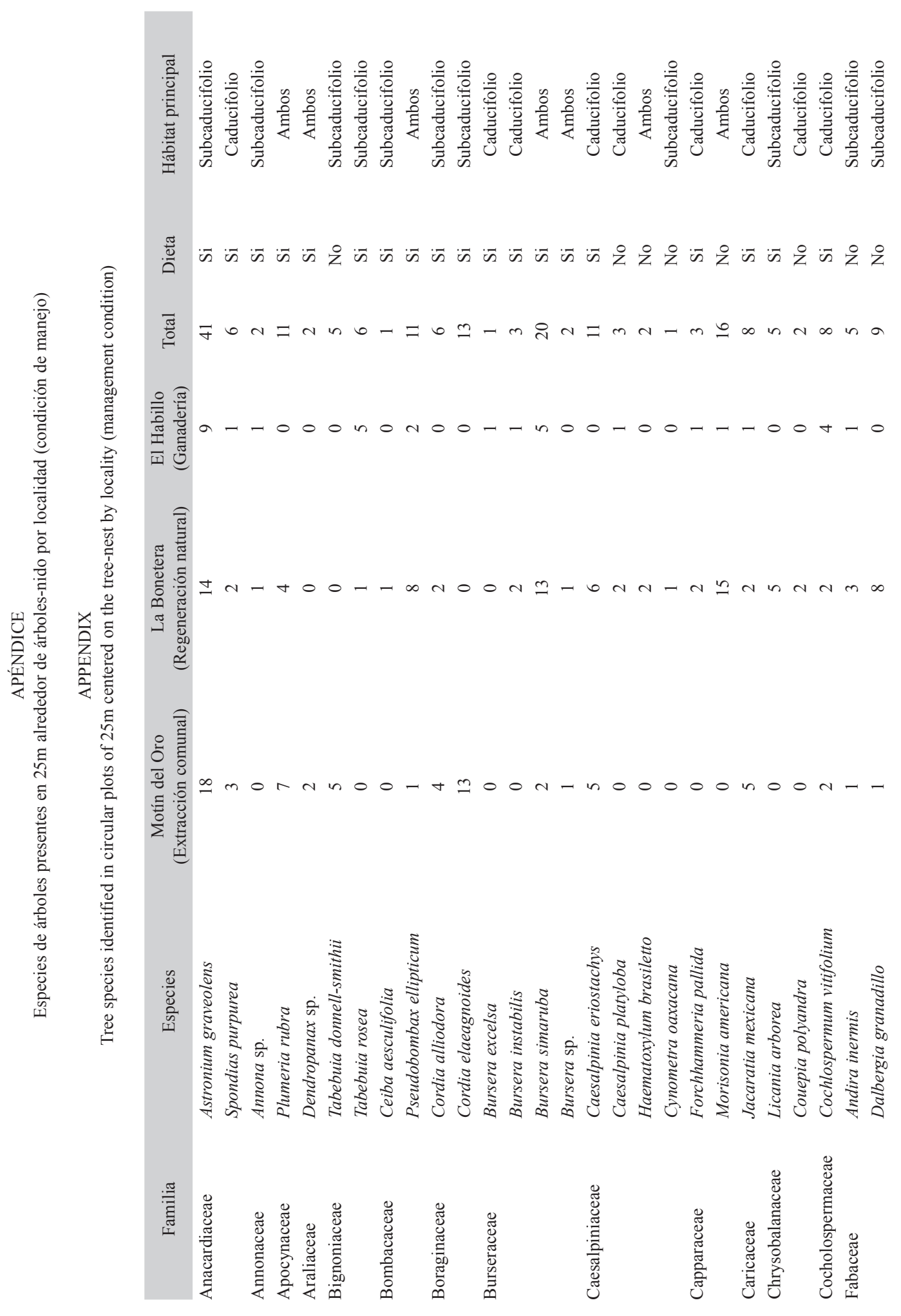




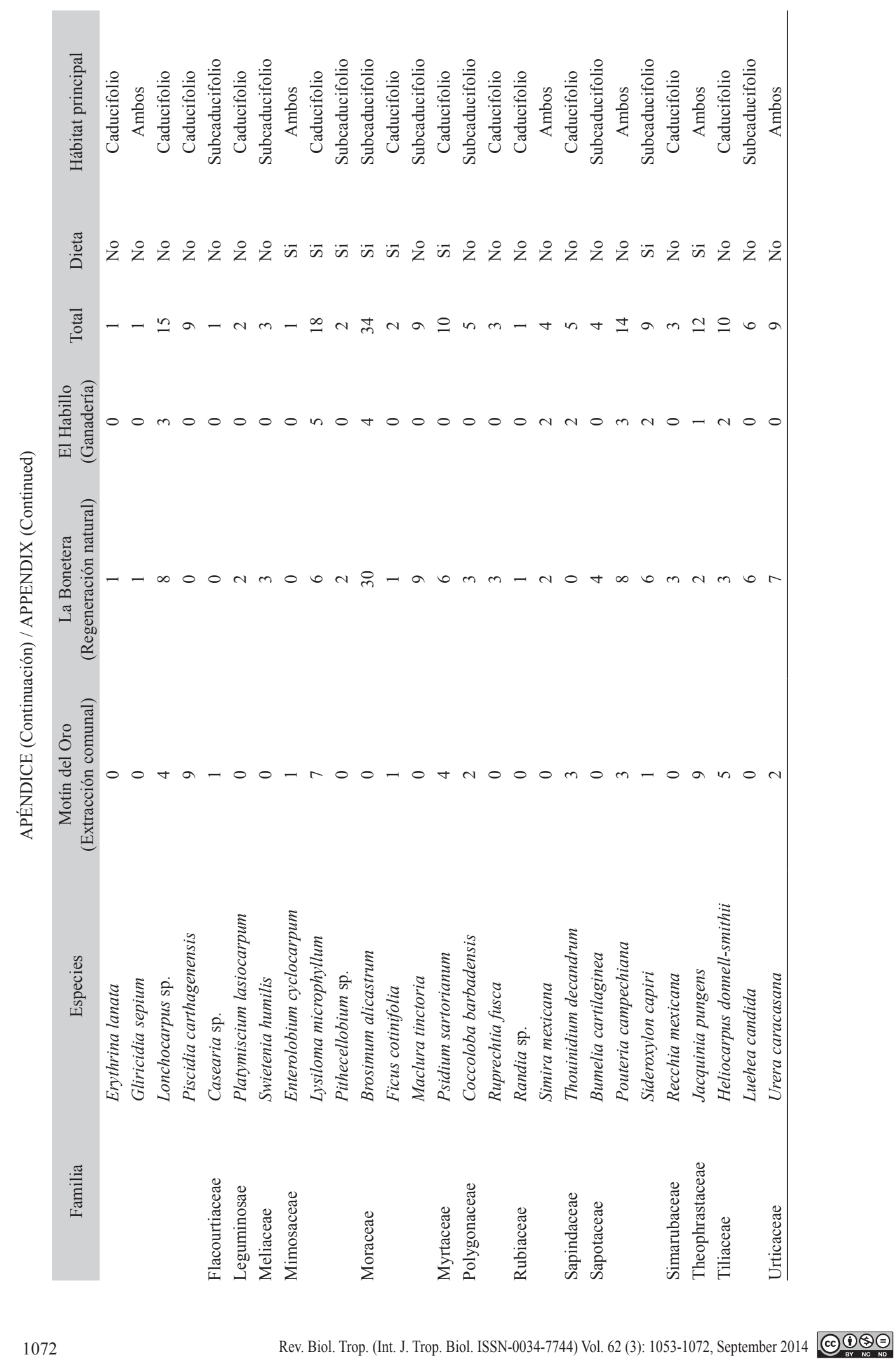

